\title{
Automaton semigroup constructions
}

\author{
Tara Brough*and Alan J. Cain ${ }^{\dagger}$
}

\begin{abstract}
The aim of this paper is to investigate whether the class of automaton semigroups is closed under certain semigroup constructions. We prove that the free product of two automaton semigroups that contain left identities is again an automaton semigroup. We also show that the class of automaton semigroups is closed under the combined operation of 'free product followed by adjoining an identity'. We present an example of a free product of finite semigroups that we conjecture is not an automaton semigroup. Turning to wreath products, we consider two slight generalizations of the concept of an automaton semigroup, and show that a wreath product of an automaton monoid and a finite monoid arises as a generalized automaton semigroup in both senses. We also suggest a potential counterexample that would show that a wreath product of an automaton monoid and a finite monoid is not a necessarily an automaton monoid in the usual sense.
\end{abstract}

\section{Introduction}

Automaton groups (that is, groups of automorphisms of labelled rooted trees generated by actions of automata) arose in the 1980s as interesting examples having 'exotic' properties. Grigorčuk's infinite periodic group [7] was the first such example, and it inspired later ones such as the Gupta-Sidki group [8]. Since then, a substantial theory has developed; see, for example, Nekrashevych's monograph [12 or one of the surveys by the school led by Bartholdi, Grigorchuk, Nekrashevych, and Šunić [1, 2, 5].

\footnotetext{
${ }^{*}$ School of Mathematics and Statistics, Mathematical Institute, North Haugh, St Andrews, Fife KY16 9SS, Scotland. Email: tara@mcs.st-andrews.ac.uk.

${ }^{\dagger}$ Centro de Matemática, Faculdade de Ciências, Universidade do Porto, Rua do Campo Alegre 687, 4169-007 Porto, Portugal. Email: ajcain@fc.up.pt
} 
In recent years, the notion of an automaton semigroup has emerged as a natural generalization of automaton groups. The basic theory was outlined by Grigorchuk, Nekrashevych \& Sushchanskii [6, esp. §4 \& §7.2]. Silva \& Steinberg studied a class of semigroup generalizing the lamplighter group [13. Maltcev studied automaton semigroups arising from Cayley automata (which arise from the Cayley graphs of finite semigroups) 10. These semigroups also formed part of studies by the second author [3] and by Mintz [11. There have also been studies of algorithmic problems (see, for instance, [9]), leading to the recent proof that the finiteness problem is undecidable for automaton semigroups [4].

A fundamental question has been whether the class of automaton semigroups is closed under various semigroup constructions. For some constructions, such as direct products and adjoining a zero or identity, it is straightforward to prove that the class is closed; see [3, $\S 5]$. This paper deals with the problems of whether the class of automaton semigroups is closed under forming semigroup or monoid free products; and whether the class automaton monoids is closed under forming wreath products with finite top semigroup. The first of these problems is connected with the even more fundamental question of whether all finite-rank free groups (which are, after all, free products of copies of the infinite cyclic group) arise as automaton groups; only recently has this question been answered positively [14]. In contrast, it is relatively easy to construct all free semigroups (of rank at least 2) as automaton semigroups [3, Proposition 4.1].

One problem with constructing counterexamples is that if a semigroup has the properties that automaton semigroups have generally, such as residual finiteness [3, Proposition 3.2], then there are no general techniques for proving it is not an automaton semigroup. For instance, the proof that the free semigroup of rank 1 is not an automaton semigroup is highly specialized and does not seem to generalize to give any useful strategy [3, Proposition 4.3]. For research on this topic to develop much further, it will be essential to develop techniques for proving that a given semigroup is not an automaton semigroup. 


\section{Automaton semigroups}

An automaton $\mathcal{A}$ is formally a triple $(Q, B, \delta)$, where $Q$ is a finite set of states, $B$ is a finite alphabet of symbols, and $\delta$ is a transformation of the set $Q \times B$. The automaton $\mathcal{A}$ is normally viewed as a directed labelled graph with vertex set $Q$ and an edge from $q$ to $r$ labelled by $x \mid y$ when $(q, x) \delta=(r, y)$ :

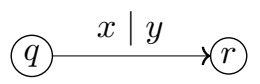

The interpretation of this is that if the automaton $\mathcal{A}$ is in state $q$ and reads symbol $x$, then it changes to the state $r$ and outputs the symbol $y$. Thus, starting in some state $q_{0}$, the automaton can read a sequence of symbols $\alpha_{1} \alpha_{2} \ldots \alpha_{n}$ and output a sequence $\beta_{1} \beta_{2} \ldots \beta_{n}$, where $\left(q_{i-1}, \alpha_{i}\right) \delta=\left(q_{i}, \beta_{i}\right)$ for all $i=1, \ldots, n$.

Such automata are more usually known in computer science as deterministic real-time (synchronous) transducers. In the field of automaton semigroups and groups, they are simply called 'automata' and this paper retains this terminology.

Each state $q \in Q$ acts on $B^{*}$, the set of finite sequences of elements of $B$. The action of $q \in Q$ on $B^{*}$ is defined as follows: $\alpha \cdot q$ (the result of $q$ acting on $\alpha$ ) is defined to be the sequence the automaton outputs when it starts in the state $q$ and reads the sequence $\alpha$. That is, if $\alpha=\alpha_{1} \alpha_{2} \ldots \alpha_{n}$ (where $\alpha_{i} \in B$ ), then $\alpha \cdot q$ is the sequence $\beta_{1} \beta_{2} \ldots \beta_{n}$ (where $\beta_{i} \in B$ ), where $\left(q_{i-1}, \alpha_{i}\right) \delta=\left(q_{i}, \beta_{i}\right)$ for all $i=1, \ldots, n$, with $q_{0}=q$.

The set $B^{*}$ can be identified with an ordered regular tree of degree $|B|$. The vertices of this tree are labelled by the elements of $B^{*}$. The root vertex is labelled with the empty word $\varepsilon$, and a vertex labelled $\alpha$ (where $\alpha \in B^{*}$ ) has $|B|$ children whose labels are $\alpha \beta$ for each $\beta \in B$. It is convenient not to distinguish between a vertex and its label, and thus one normally refers to 'the vertex $\alpha$ ' rather than 'the vertex labelled by $\alpha$ '. (Figure 1 illustrates the tree corresponding to $\{0,1\}^{*}$.)

The action of a state $q$ on $B^{*}$ can thus be viewed as a transformation of the corresponding tree, sending the vertex $w$ to the vertex $w \cdot q$. Notice that, by the definition of the action of $q$, if $\alpha \alpha^{\prime} \cdot q=\beta \beta^{\prime}$ (where $\alpha, \beta \in B^{*}$ and $\alpha^{\prime}, \beta^{\prime} \in B$ ), then $\alpha \cdot q=\beta \cdot q$. In terms of the transformation on the tree, 


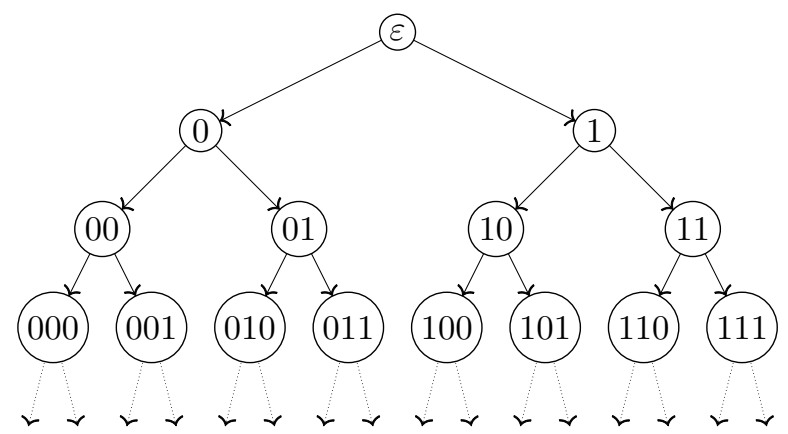

Figure 1: The set $\{0,1\}^{*}$ viewed as a rooted binary tree.

this says that if one vertex $(\alpha)$ is the parent of another $\left(\alpha \alpha^{\prime}\right)$, then their images under the action by $q$ are also parent $(\beta)$ and child $\left(\beta \beta^{\prime}\right)$ vertices. More concisely, the action of $q$ on the tree preserves adjacency and is thus an endomorphism of the tree. Furthermore, the action's preservation of lengths of sequences becomes a preservation of levels in the tree.

The actions of states extends naturally to actions of words: $w=w_{1} \cdots w_{n}$ (where $w_{i} \in Q$ ) acts on $\alpha \in B^{*}$ by

$$
\left(\cdots\left(\left(\alpha \cdot w_{1}\right) \cdot w_{2}\right) \cdots w_{n-1}\right) \cdot w_{n} .
$$

So there is a natural homomorphism $\phi: Q^{+} \rightarrow$ End $B^{*}$, where $\operatorname{End} B^{*}$ denotes the endomorphism semigroup of the tree $B^{*}$. The image of $\phi$ in End $B^{*}$, which is necessarily a semigroup, is denoted $\Sigma(\mathcal{A})$.

A semigroup $S$ is called an automaton semigroup if there exists an automaton $\mathcal{A}$ such that $S \simeq \Sigma(\mathcal{A})$.

It is often more convenient to reason about the action of a state or word on a single sequence of infinite length than on sequences of some arbitrary fixed length. The set of infinite sequences over $B$ is denoted $B^{\omega}$. The infinite sequence consisting of countably many repetitions of the finite word $\alpha \in B^{*}$ is denoted $\alpha^{\omega}$. For synchronous automata, the action on infinite sequences determines the action on finite sequences and vice versa.

The following lemma summarizes the conditions under which two words $w$ and $w^{\prime}$ in $Q^{+}$represent the same element of the automaton semigroup. The results follow immediately from the definitions, but are so fundamental that they deserve explicit statement: 
Lemma 1. Let $w, w^{\prime} \in Q^{+}$. Then the following are equivalent:

(i) $w$ and $w^{\prime}$ represent the same element of $\Sigma(\mathcal{A})$;

(ii) $w \phi=w^{\prime} \phi$;

(iii) $\alpha \cdot w=\alpha \cdot w^{\prime}$ for each $\alpha \in B^{*}$;

(iv) $w$ and $w^{\prime}$ have the same actions on $B^{n}$ for every $n \in \mathbb{N}^{0}$;

(v) $w$ and $w^{\prime}$ have the same actions on $B^{\omega}$.

Generally, there is no need to make a notational distinction between $w$ and $w \phi$. Thus $w$ denotes both an element of $Q^{+}$and the image of this word in $\Sigma(\mathcal{A})$. In particular, one writes ' $w=w^{\prime}$ in $\Sigma(\mathcal{A})$ ' instead of the strictly correct ' $w \phi=w^{\prime} \phi$ '. With this convention, notice that $Q$ generates $\Sigma(\mathcal{A})$.

\section{$3 \quad$ Free products}

The semigroup free product (henceforth free product) of two semigroups $S=$ $\operatorname{Sgp}\left\langle X_{1} \mid R_{1}\right\rangle$ and $T=\operatorname{Sgp}\left\langle X_{2} \mid R_{2}\right\rangle$, denoted $S \star T$, is the semigroup with presentation $\operatorname{Sgp}\left\langle X_{1} \cup X_{2} \mid R_{1} \cup R_{2}\right\rangle$. If $S=\operatorname{Mon}\left\langle X_{1} \mid R_{1}\right\rangle$ and $T=\operatorname{Mon}\left\langle X_{2} \mid R_{2}\right\rangle$ are monoids, then the monoid free product $S *_{\text {Mon }} T$ of $S$ and $T$ is the monoid with presentation $\operatorname{Mon}\left\langle X_{1} \cup X_{2} \mid R_{1} \cup R_{2}\right\rangle$. The difference is that the identities of $S$ and $T$ are amalgamated in the monoid free product, but not in the semigroup free product (even if present).

We will show that if two automaton semigroups both contain left identities, then their free product is an automaton semigroup. Also, if we take the free product of any two automaton semigroups and adjoin an identity, then the result is an automaton semigroup. We conjecture that the class of automaton semigroups is not closed under taking free products, and we suggest a possible counterexample.

Theorem 2. Let $S$ and $T$ be automaton semigroups, each containing a left identity. Then $S \star T$ is an automaton semigroup.

[There are two dual notions of automaton semigroups, depending on whether the states act on the right (as in this paper) or on the left. Using the dual 
notion would give a version of this proposition for semigroups with right identities.]

Proof. Let $l_{S}$ and $l_{T}$ be distinguised left identities in $S$ and $T$ respectively. Let $\mathcal{A}_{1}=\left(Q_{1}, A, \delta_{1}\right)$ and $\mathcal{A}_{2}=\left(Q_{2}, B, \delta_{2}\right)$ be automata with $\Sigma\left(\mathcal{A}_{1}\right)=S$ and $\Sigma\left(\mathcal{A}_{2}\right)=T$. We may assume that $Q_{1} \subset S$ and $Q_{2} \subset T$ and that $Q_{1}$ and $Q_{2}$ contain $l_{S}$ and $l_{T}$ respectively.

We construct an automaton $\mathcal{A}$ with $\Sigma(\mathcal{A})=S \star T$ using $\mathcal{A}_{1}$ and $\mathcal{A}_{2}$. First let $A^{\circ}=\left\{a^{\circ} \mid a \in A\right\}$ and $B^{\circ}=\left\{b^{\circ} \mid b \in B\right\}$. Let $\mathcal{A}=(Q, C, \delta)$ with $Q=Q_{1} \cup Q_{2}, C=A \cup B \cup A^{\circ} \cup B^{\circ} \cup\left\{\$, \#, \$^{\circ}, \#^{\circ}\right\}$ and $\delta: Q \times C \rightarrow Q \times C$ given by $\delta\left(q, x^{\circ}\right)=\left(q, x^{\circ}\right)$ for all $q \in Q$ and $x \in A \cup B \cup\{\$, \#\}$ and

$$
\begin{aligned}
\delta(s, a) & =\delta_{1}(s, a) & \delta(t, b) & =\delta_{2}(t, b) \\
\delta(s, b) & =\left(s, b^{\circ}\right) & \delta(t, a) & =\left(t, a^{\circ}\right) \\
\delta(s, \#) & =\left(s, \#^{\circ}\right) & \delta(t, \$) & =\left(t, \$^{\circ}\right) \\
\delta(s, \$) & =\left(l_{T}, \$\right) & \delta(t, \#) & =\left(l_{S}, \#\right)
\end{aligned}
$$

for $a \in A, b \in B, s \in Q_{1}$ and $t \in Q_{2}$.

We will henceforth refer to the subautomaton consisting of states from $Q_{i}$ as $\mathcal{A}_{i}^{\prime}(i=1,2)$. We will refer to symbols in $A^{\circ} \cup B^{\circ} \cup\left\{\$^{\circ}, \#^{\circ}\right\}$ as 'marked symbols'. All states act trivially on marked symbols. Symbols are marked in order to show that they are finished with and should never be altered further.

The subautomaton $\mathcal{A}_{1}^{\prime}$ acts the same as $\mathcal{A}_{1}$ on symbols from $A$, marks symbols from $B \cup\{\#\}$ without changing state, and transitions to the state $l_{T}$ in $\mathcal{A}_{2}$ on input $\$$, outputting $\$$. The construction is symmetric in the pairs $\left(\mathcal{A}_{1}, \mathcal{A}_{2}\right),\left(\mathcal{A}_{1}^{\prime}, \mathcal{A}_{2}^{\prime}\right),(A, B)$ and $(\$, \#)$.

Any $\alpha \in C^{*}$ is a prefix of some $\beta=u_{1} \$ v_{1} \# \ldots u_{i} \$ v_{i} \# \ldots \in C^{\omega}$ with $u_{j} \in(C \backslash\{\$\})^{*}$ and $v_{j} \in(C \backslash\{\#\})^{*}$. We have

$$
\beta \cdot l_{S}=\left(u_{1} \cdot l_{S}\right) \$\left(v_{1} \cdot l_{T}\right) \# \ldots\left(u_{i} \cdot l_{S}\right) \$\left(v_{i} \cdot l_{T}\right) \# \ldots
$$

Since $l_{S}$ and $l_{T}$ act as idempotents on $C \backslash\{\$\}$ and $C \backslash\{\#\}$ respectively, this shows that $l_{S}$ (and, by symmetry, $l_{T}$ ) is an idempotent in $\Sigma(\mathcal{A})$. Hence, for $\beta$ as above and $s_{1} \ldots s_{k} \in Q_{1}$, we have

$$
\begin{aligned}
\beta \cdot s_{1} \ldots s_{k} & =\left(u_{1} \cdot s_{1}\right) \$\left(v_{1} \cdot l_{T}\right) \# \ldots\left(u_{i} \cdot l_{S}\right) \$\left(v_{i} \cdot l_{T}\right) \# \ldots \cdot s_{2} \ldots s_{k} \\
& =\left(u_{1} \cdot s_{1} \ldots s_{k}\right) \$\left(v_{1} \cdot l_{T}\right) \# \ldots\left(u_{i} \cdot l_{S}\right) \$\left(v_{i} \cdot l_{T}\right) \# \ldots,
\end{aligned}
$$


so the action of $Q_{1}^{+}$on $C^{*}$ depends only on its action on $A^{*}$, and hence the subsemigroup of $\Sigma(\mathcal{A})$ generated by $Q_{1}^{+}$is isomorphic to $S$. By symmetry, the subsemigroup generated by $Q_{2}^{+}$is isomorphic to $T$. Hence, by induction, any two words in $Q^{+}$representing the same element of $S \star T$ will act the same on $C^{*}$, so $\mathcal{A}$ defines an action of $S \star T$ on $C^{*}$.

Now in order to conclude that $S(\mathcal{A})=S \star T$, we need to show that the action of $S \star T$ on $C^{\omega}$ defined by $\mathcal{A}$ is faithful. We can distinguish most pairs of strings by their actions on $(\$ \#)^{\omega}$ and $(\# \$)^{\omega}$. For $w \in Q^{+}$, define $x_{w}=(\$ \#)^{\omega} \cdot w$ and $y_{w}=(\# \$)^{\omega} \cdot w$. Let $\bar{w}$ be the reduced word in $(S \cup T)^{*}$ corresponding to $w$. For $w \in Q_{1} Q^{+}$, we have

$$
x_{w}= \begin{cases}\left(\$^{\circ} \#^{\circ}\right)^{k-1} \$^{\circ}(\# \$)^{\omega} & \text { if } l(\bar{w})=2 k \\ \left(\$^{\circ} \#^{\circ}\right)^{k}(\$ \#)^{\omega} & \text { if } l(\bar{w})=2 k+1\end{cases}
$$

while

$$
y_{w}= \begin{cases}\left(\#^{\circ} \$^{\circ}\right)^{k}(\# \$)^{\omega} & \text { if } l(\bar{w})=2 k, \\ \left(\#^{\circ} \$^{\circ}\right)^{k} \#^{\circ}(\$ \#)^{\omega} & \text { if } l(\bar{w})=2 k+1 .\end{cases}
$$

Together with the corresponding statements for $w \in Q_{2} Q^{+}$, this tells us that the only pairs elements of $S \star T$ which cannot be distinguished by $x_{w}$ and $y_{w}$ are those of the same reduced length, beginning with a letter from the same $Q_{i}$.

Finally, let $w$ and $w^{\prime}$ be words in $Q^{+}$of the same reduced length, representing different elements of $S \star T$ and starting with symbols from the same $Q^{+}$. The cases for odd and even length are almost identical; we present the even length case. Without loss of generality, take $w=s_{1} t_{1} \ldots s_{k} t_{k}$ and $w^{\prime}=s_{1}^{\prime} t_{1}^{\prime} \ldots s_{k}^{\prime} t_{k}^{\prime}$ with $s_{i}, s_{i}^{\prime} \in Q_{1}^{+}$and $t_{i}, t_{i}^{\prime} \in Q_{2}^{+}$. If $w$ and $w^{\prime}$ represent different elements of $S \star T$, then we must have $\overline{s_{i}} \neq \overline{s_{i}^{\prime}}$ or $\overline{t_{i}} \neq \overline{t_{i}^{\prime}}$ for some $i$. Let us suppose the former, the other case being very similar. Let $\alpha \in A^{*}$ with $\alpha \cdot s_{i} \neq \alpha \cdot s_{i}^{\prime}$ and let $\beta=(\$ \#)^{i-1} \alpha(\$ \#)^{k-i+1}$. Then

$$
\begin{aligned}
& \beta \cdot w=\left(\$^{\circ} \#^{\circ}\right)^{i-1}\left(\alpha \cdot s_{i}\right)^{\circ}\left(\$^{\circ} \#^{\circ}\right)^{k-i} \$^{\circ} \#, \\
& \beta \cdot w^{\prime}=\left(\$^{\circ} \#^{\circ}\right)^{i-1}\left(\alpha \cdot s_{i}^{\prime}\right)^{\circ}\left(\$^{\circ} \#^{\circ}\right)^{k-i} \$^{\circ} \#
\end{aligned}
$$

and so $w \neq w^{\prime}$ in $\Sigma(\mathcal{A})$. Hence $\Sigma(\mathcal{A})=S \star T$.

The next theorem shows that free products of automaton semigroups are very close to being automaton semigroups. 
Theorem 3. Let $S$ and $T$ be automaton semigroups. Then $(S \star T)^{1}$ is an automaton semigroup.

Proof sketch. The idea is very similar to the previous proposition, but instead of the states $1_{S}$ and $1_{T}$ we have a single state 1 , which acts as the identity on all strings.

We construct an automaton $\mathcal{A}$ with $\Sigma(\mathcal{A})=(S \star T)^{1}$ as follows: Let $\mathcal{A}_{1}=$ $\left(Q_{1}, A, \delta_{1}\right)$ and $\mathcal{A}_{2}=\left(Q_{2}, B, \delta_{2}\right)$ be automata for $S$ and $T$ respectively and let the alphabet of $\mathcal{A}$ be $C=A \cup B \cup A^{\circ} \cup B^{\circ} \cup\left\{\$, \#, \$^{\circ}\right.$, $\left.\#^{\circ}\right\}$, with $A^{\circ}$ and $B^{\circ}$ defined as in the previous proof. Let the set of states of $\mathcal{A}$ be $S \cup T \cup\{1\}$. Define the transition function $\delta: Q \times C \rightarrow Q \times C$ by $\delta(1, c)=(1, c)$ for all $c \in C, \delta\left(q, x^{\circ}\right)=\left(q, x^{\circ}\right)$ for all $q \in Q, x \in A \cup B \cup\{\$, \#\}$ and

$$
\begin{aligned}
\delta(s, a) & =\delta_{1}(s, a) & \delta(t, b) & =\delta_{2}(t, b) \\
\delta(s, b) & =\left(s, b^{\circ}\right) & \delta(t, a) & =\left(t, a^{\circ}\right) \\
\delta(s, \#) & =\left(s, \#^{\circ}\right) & \delta(t, \$) & =\left(t, \#^{\circ}\right) \\
\delta(s, \$) & =(1, \$) & \delta(t, \#) & =(1, \#)
\end{aligned}
$$

for $a \in A, b \in B, s \in Q_{1}, t \in Q_{2}$.

As before, the actions of $S$ on $(C \backslash\{\$\})^{*}$ and of $T$ on $(C \backslash\{\#\})^{*}$ are determined solely by their actions on $A^{*}$ and $B^{*}$ respectively. For $u \in(C \backslash\{\$\})^{*}$, $v \in C^{\omega}$ and $s \in Q_{1}^{+}$, we have $u \$ v \cdot s=(u \cdot s) \$ v$. So again the subsemigroups generated by $Q_{1}$ and by $Q_{2}$ are isomorphic to $S$ and $T$ respectively, and since the state 1 acts as the identity on all strings, we conclude that $\mathcal{A}$ defines an action of $(S \star T)^{1}$ on $C^{*}$.

We distinguish between elements of $(S \star T)^{1}$ in essentially the same way as in the previous proof.

If $S$ and $T$ are semigroups, then $S^{1} *_{\text {Mon }} T^{1}=(S \star T)^{1}$. If $M$ is a monoid with indecomposable identity, then $S=M \backslash\{1\}$ is a semigroup with $M=S^{1}$. Hence we have the following corollary to Proposition 3 .

Corollary 4. The monoid free product of two automaton monoids with indecomposable identities is an automaton monoid.

Our constructions rely heavily on the use of a state acting as an identity or left identity on all strings. Since all free semigroups of rank at least 2 are 
automaton semigroups, we know that the presence of left identities is not essential for a free product of automaton semigroups to be an automaton semigroup, but we conjecture that the class of automaton semigroups is not closed under free products. Indeed we conjecture something a good deal stronger:

Conjecture 5. There exist finite semigroups $S$ and $T$ such that $S \star T$ is not an automaton semigroup.

We have some hope that we may be able to prove this conjecture using very small semigroups $S$ and $T$, for example $S$ the trivial semigroup and $T$ a two-element null semigroup (that is, $T=\{t, z\}$ with all products equal to $z)$.

\section{Wreath products}

The wreath product of two automaton semigroups is certainly not always an automaton semigroup, since it need not even be finitely generated. One way to ensure that a wreath product $S \imath T$ is finitely generated is to require $S$ and $T$ to be monoids, with $T$ finite. The second author asked in [3] whether, under these restrictions, taking wreath products of automaton monoids always gives an automaton monoid. The answer is almost certainly no, and in fact wreath products of automaton monoids are probably almost never automaton monoids.

For monoids $S$ and $T$ with $T=\left\{t_{1}, \ldots, t_{n}\right\}$ finite, the wreath product $S \nmid T$ of $S$ with $T$ is a semidirect product $S^{|T|} \rtimes T$, where $T$ acts on elements of $S^{|T|}$ by $\left(s_{t_{1}}, s_{t_{2}}, \ldots, s_{t_{n}}\right)^{t}=\left(s_{t_{1} t}, s_{t_{2} t}, \ldots, s_{t_{n}}\right)$.

Conjecture 6. The wreath product $\mathbb{N}_{0} \succ C_{2}$ is not an automaton monoid.

The obstruction to constructing an automaton $\mathcal{A}$ with $\Sigma(\mathcal{A})=S \imath T$ seems to be that the automaton needs to be aware at all times of the 'current element' of $T$, in order for states from $S$ to act correctly. By this we mean that if we are computing the action of a word $q_{1} \ldots q_{n}$, then in order to know how $q_{i}$ should act on strings, if $q_{1} \ldots q_{i-1}=s_{i} t_{i}$ with $s_{i} \in S, t_{i} \in T$, then we need to know $t_{i}$. If we attempt to store this information in the states, we end up with states which do not act as elements of $S \imath T$ on strings. If we attempt to store the information in the strings, the difficulty is that we would like 
it to occur only once, at the start of the string, but it seems impossible to guarantee this without using additional states. The automaton cannot tell what point it is up to in the string, so it treats all symbols of the type used to encode the current $t_{i}$ as if they were in fact $t_{i}$, leading seemingly unavoidably to 'misdirections', in which the automaton does not act as intended.

Semigroups generated by only some of the states in an automaton are also worthwhile objects of study, considered for example in [6]. They are, of course, simply finitely generated subsemigroups of automaton semigroups (since we can always add any finite set of elements of a semigroup to the state set for the automaton). With this point of view, our first obstruction to closure under wreath products falls away.

Theorem 7. Let $S$ and $T$ be automaton monoids with $T$ finite. Then $S \imath T$ is a finitely generated subsemigroup of an automaton semigroup.

Proof. Let $S=\Sigma(\mathcal{A})$ with $\mathcal{A}=(Q, A, \delta)$ be an automaton monoid and let $T=\left\{t_{1}, \ldots, t_{n}\right\}$ be a finite monoid. We construct an automaton $\mathcal{B}$ such that $S \imath T$ is a subsemigroup of $\Sigma(\mathcal{B})$. The automaton $\mathcal{B}$ has state set $Q_{1}^{n} \cup Q_{2}^{n} \cup T$, where each $Q_{i}$ is a copy of $Q$; and alphabet $A^{n} \cup B$, where $B$ is a copy of $T$. For $q \in Q^{n}$, we denote the copy of $q$ in $Q_{i}$ by $q_{i}$.

In a state $t \in T$, the automaton remains in state $T$, not altering the input string until a symbol $b \in B$ is read, at which point it outputs $\overline{b t}$ and moves to state $1_{T}$, hence leaving the remainder of the string unchanged. The states in $Q_{2}^{n}$ act on symbols in $A^{n}$ in exactly the same way as in the standard automaton for the direct product $S^{n}$ (see [3, Proposition 5.5]), ignoring symbols from $B$. A state $s \in Q_{1}^{n}$ ignores symbols from $A^{n}$, and moves to the state corresponding to $s^{b}$ in $Q_{2}^{n}$ upon reading a symbol $b \in B$ (leaving $b$ unchanged).

All symbols from $A^{n}$ before the first symbol from $B$, and all subsequent symbols from $B$, are ignored by all states. Hence the action of $\mathcal{B}$ on $\left(A^{n} \cup B\right)^{*}$ is completely determined by its action on $B\left(A^{n}\right)^{\omega}$. For $\alpha \in\left(A^{n}\right)^{*}, b \in B$, $q_{i} \in Q_{i}^{n}(i=1,2)$ and $t \in T$, we have

$$
b \alpha \cdot q_{1}=b\left(\alpha \cdot q^{b}\right), b \alpha \cdot q_{2}=b(\alpha \cdot q) \text { and } b \alpha \cdot t=\overline{b t} \alpha .
$$

Using these facts, it is straightforward to show that $S<T$ is isomorphic to the subsemigroup of $\Sigma(\mathcal{A})$ generated by $Q_{1}^{n} \cup T$. 
We might choose to remove the second obstruction instead, by allowing ourselves to restrict the set of strings the automaton acts on. The proof of Theorem 7 gives an indication of one way to do this. We define an initialsymbol automaton semigroup to be a semigroup which is obtained from an automaton $\mathcal{A}=(Q, B, \delta)$ in the same way as the automaton semigroup $\Sigma(\mathcal{A})$, except that we only consider the action on strings in $C D^{\omega}$, where $B$ is the disjoint union of $C$ and $D$. This is not so far from an automaton semigroup, as $C D^{\omega}$ can still be viewed as a rooted (almost regular) tree, but with the root having a (potentially) different degree to the remaining vertices. Such a tree is a very natural structure for a wreath product to act on.

Theorem 8. Let $S$ and $T$ be automaton monoids with $T$ finite. Then $S \imath T$ is an initial-symbol automaton monoid.

Proof. If in the proof of the previous theorem, we restrict the automaton $\mathcal{B}$ to act only on strings in $B A^{\omega}$, then one copy of $Q^{n}$ suffices in the state set, since the purpose of the first copy was simply to record whether we have yet encountered a symbol from $B$ in processing the string. We can thus obtain $S \imath T$ as an initial-automaton monoid by using an automaton similar to the automaton $\mathcal{B}$ in the previous proof, except that it has states $Q^{n}$ in place of $Q_{1}^{n} \cup Q_{2}^{n}$, and these states act like their corresponding versions in $Q_{1}^{n}$ on $B$, and like their corresponding versions in $Q_{2}^{n}$ on $A$.

It may be worth considering further what kind of restrictions on the strings acted on by an automaton give rise to interesting classes of semigroups.

\section{$5 \quad$ Further constructions}

A semigroup $S$ is a small extension of another semigroup $T$ if $T \leq S$ and $|S \backslash T|$ is finite. It is easy to see that in this case $S$ being an automaton semigroup need not imply that $T$ is an automaton semigroup, since the free monoid of rank 1 is an automaton semigroup [3, Proposition 4.4], while the free semigroup of rank 1 is not [3, Proposition 4.3]. The other direction remains open:

Open Problem 9. [3], Open Problem 5.4] If a semigroup $S$ is a small extension of an automaton semigroup $T$, is $S$ necessarily an automaton semigroup? 
We again expect the answer to be no. One possible counterexample is a certain strong semilattice of two semigroups, with the 'lower' semigroup being finite. Some other open problems include the closure or otherwise of the class of automaton semigroups under Rees matrix constructions, and whether if $S$ is a semigroup such that adjoining a zero to $S$ results in an automaton semigroup, then $S$ itself must be an automaton semigroup.

\section{Acknowledgements}

The first author's research was funded by an EPSRC grant EP/H011978/1.

The second author's research was funded by the European Regional Development Fund through the programme COMPETE and by the Portuguese Government through the FCT (Fundação para a Ciência e a Tecnologia) under the project PEsT-C/MAT/UI0144/2011 and through an FCT Ciência 2008 fellowship.

\section{References}

[1] L. Bartholdi, R. Grigorchuk, and V. Nekrashevych. From fractal groups to fractal sets. In Fractals in Graz 2001, Trends Math., pages 25-118. Birkhäuser, Basel, 2003.

[2] L. Bartholdi, R. I. Grigorchuk, and Z. Šuniḱ. Branch groups. In Handbook of Algebra, Vol. 3, pages 989-1112. North-Holland, Amsterdam, 2003.

[3] A. J. Cain. Automaton semigroups. Theoret. Comput. Sci., 410(4749):5022-5038, 2009.

[4] P. Gillibert. The finiteness problem for automaton semigroups is undecidable. Preprint, arXiv:1304.2295.

[5] R. Grigorchuk and Z. Šunić. Self-similarity and branching in group theory. In Groups St. Andrews 2005. Vol. 1, volume 339 of London Math. Soc. Lecture Note Ser., pages 36-95. Cambridge Univ. Press, Cambridge, 2007. 
[6] R. I. Grigorchuk, V. V. Nekrashevich, and V. I. Sushchanskiiu. Automata, dynamical systems, and groups. Proc. Steklov Inst. Math., 231(4):128-203, 2000.

[7] R. I. Grigorčuk. On Burnside's problem on periodic groups. Funktsional. Anal. i Prilozhen., 14(1):53-54, 1980.

[8] N. Gupta and S. Sidki. On the Burnside problem for periodic groups. Math. Z., 182(3):385-388, 1983.

[9] I. Klimann, J. Mairesse, and M. Picantin. Implementing computations in automaton (semi)groups. In N. Moreira and R. Reis, editors, Implementation and Application of Automata, volume 7381 of Lecture Notes in Computer Science, pages 240-252. Springer, Berlin, 2012.

[10] V. Maltcev. Cayley automaton semigroups. Internat. J. Algebra Comput., 19(1):79-95, 2009.

[11] A. Mintz. On the Cayley semigroup of a finite aperiodic semigroup. Internat. J. Algebra Comput., 19(6):723-746, 2009.

[12] V. Nekrashevych. Self-similar groups, volume 117 of Mathematical Surveys and Monographs. American Mathematical Society, Providence, RI, 2005.

[13] P. V. Silva and B. Steinberg. On a class of automata groups generalizing lamplighter groups. Internat. J. Algebra Comput., 15(5-6):1213-1234, 2005.

[14] B. Steinberg, M. Vorobets, and Y. Vorobets. Automata over a binary alphabet generating free groups of even rank. Internat. J. Algebra Comput., 21(1-2):329-354, 2011. 